\title{
DUALITY THEORY FOR LOCALLY COMPACT GROUPS WITH PRECOMPACT CONJUGACY CLASSES. II: THE DUAL SPACE
}

\author{
BY
}

TERJE SUND( ${ }^{1}$ )

\begin{abstract}
The present paper is concerned with the dual space $\hat{G}$ consisting of all unitary equivalence classes of continuous irreducible unitary representations of separable $[F C]^{-}$groups (i.e. groups with precompact conjugacy classes). The main purpose of the paper is to extend certain results from the duality theory of abelian groups and $[Z]$ groups to the larger class of $[F C]^{-}$ groups. In addition, we deal briefly with square-integrability for representations of $[F C]^{-}$groups. Most of our results are proved for type I groups. Our key result is that $\hat{G}$ may be written as a disjoint union of abelian topological $T_{4}$ groups, which are open in $\hat{G}$.
\end{abstract}

Introduction. In [23] we studied the character space $X(G)$ of locally compact groups possessing precompact conjugacy classes ( $[F C]^{-}$groups), and we were able to extend much of the classical duality theory for abelian groups(2) to this larger class of groups using $\mathfrak{X}(G)$ as dual object. (3) The present paper is concerned with the dual space $\hat{G}$ of all unitary equivalence classes of continuous irreducible unitary Hilbert space representations of separable $[F C]^{-}$groups $G$. $\hat{G}$ is given the Fell topology, obtained from the hull kernel topology on the primitive ideal space of the group $C^{*}$-algebra [4].

For an arbitrary locally compact group $G$, insight into the structure of $\hat{G}$ often tells more about the group itself than the corresponding knowledge of $\mathfrak{X}(G)$. This is partly due to the fact that the elements of $\hat{G}$ always separate the points of $G$, whereas a corresponding property (4) holds for $X(G)$ if $G \in$ $[S I N]$ but not generally. Even in the case of a type $\left.I^{[F C}\right]^{-}$group the character

Received by the editors June 20, 1974 and, in revised form, October 2, 1975.

AMS (MOS) subject classifications (1970). Primary 22D35; Secondary 43A40, 22 D10.

(1) Research partially supported by The Norwegian Research Council for Science and the Humanities, and by research funds of the Graduate School of the University of Minnesota. Part of the contents of this paper is taken from the author's doctoral dissertation at the University of Minnesota, written under Professor S. Grosser (1973).

(2) Such a generalization was carried out in [9] for the class of locally compact groups $G$ such that $G$ modulus its center is compact.

( $\left.{ }^{3}\right) X(G)$ consists of the nonzero extreme points of the set of all continuous positive definite $G$-invariant functions $f$ on $G$ such that $f(e)<1$; and $x(G)$ was endowed with the topology of uniform convergence on compacta.

(4)I.e., if $x \in G, x \neq e$, then there is a character $\lambda \in x(G)$ such that $\lambda(x) \neq 1$. 
space may constitute only a small part of the dual. For $\mathfrak{X}(G)$ is then isomorphic to the dual space $\widehat{G / C}$ of the $[F C]^{-} \cap[S I N]$ quotient group $G / C$, where $C$ denotes the intersection of all closed invariant neighborhoods of $e$ in $G$ [18, Theorem 5.12]. We shall prove, e.g., that $\hat{G}$ is connected if and only if $G$ is aperiodic (i.e., $G$ contains no nontrivial compact subgroup). For $\mathcal{X}(G)$ this result can hold only when $G$ is replaced by $G / C$; see $[23,(2.12)]$.

On the other hand, the character space $\chi(G)$ is a locally compact Hausdorff space for $G \in[F C]^{-}[12]$, whereas $\hat{G}$ need not even be a $T_{0}$ space (but $\hat{G}$ is always locally compact $[4,3.3 .8])$. In fact, $\hat{G}$ is $T_{0}$ iff $G$ is type $I$ iff $\hat{G}$ is $T_{4}$ (1.3). Whenever our method of proof utilizes topological separation properties of $\hat{G}$ it is therefore necessary (and sufficient) to assume that $G$ is type I. Under this hypothesis we can show that $\hat{G}$ is locally Euclidean iff $G$ is compactly generated, and $\hat{G}$ is locally connected iff $G$ possesses property (L).

In addition to extending classical duality results like the ones mentioned above, this paper also briefly deals with square-integrable representations of $[F C]^{-}$ groups and we provide simple, necessary and sufficient conditions for $[\pi] \in \hat{G}$ to be square-integrable.

For convenience we shall list the classes of groups with which we are dealing.

[SIN]-The class of locally compact groups possessing a fundamental system of invariant neighborhoods of $e$.

$[F C]^{-}$-The class of locally compact groups possessing precompact conjugacy classes.

$[F I A]^{-}-$The locally compact groups with precompact inner automorphism group. One has $[F I A]^{-}=[S I N] \cap[F C]^{-}$; see $[10]$.

$[F D]^{-}-$The locally compact groups with precompact commutator group.

The following inclusions hold: $[F I A]^{-} \subseteq[F C]^{-}$and $[F D]^{-} \subseteq[F C]^{-}$ [10]. Of course, all the classes of groups listed above contain the abelian groups and the compact groups.

If $\rho$ is a unitary representation of a closed subgroup $H$ of a locally compact group $G$ we shall let $\operatorname{Ind}_{H}^{G}(\rho)$ denote the unitary representation of $G$ induced from $\rho$. If $\pi$ is a representation of $G, \pi_{H}$ will denote the representation of the subgroup $H$ given by restriction, $\pi_{H}(h)=\pi(h)$, all $h \in H$. Let $\pi$ and $\sigma$ be unitary representations of $G$. We shall write $\pi \simeq \sigma$ if they are unitarily equivalent. If the topological groups $G$ and $L$ are isomorphic, we shall use the notation $G \approx$ $L$. If $\pi \in \operatorname{Irr}(G)[4,18.5 .1]$ we let $[\pi]$ denote the equivalence class of $\pi$ in $\hat{G}$. For $S \subset \operatorname{Irr}(G)$ we let $[S]$ be the image of $S$ in $\hat{G}$ under the canonical map $\pi$ $\mapsto[\pi]$. Finally, we let $\rho^{\prime}$ denote the representation of $G$ given by $\rho^{\prime}(x)=$ $\rho(x N)$ whenever $\rho$ is a representation of a factor group $G / N$.

We would like to mention at this point that E. Kaniuth recently obtained results related to the present ones under somewhat different conditions on the 
groups (Topology in duals of SIN-groups, Math. Z. 134 (1973), 67-80).

We thank the referee for several pertinent remarks, which led to improvements of the paper.

1. Groups with abelian quotients. Let $G$ be a separable locally compact group of type $I$ and suppose that $N$ is a closed normal subgroup of $G$ such that $G / N$ is abelian. Assume also that $N$ is type I and regularly embedded in $G$. In this section we shall analyze the dual of such groups. We shall assume the reader is familiar with Mackey's little group method, [1] and [17]. The main reason for the above hypothesis on $G$ is that $[F C]^{-}$groups, which are our main concern in this paper, satisfy exact sequences of topological groups $(e) \rightarrow P \rightarrow G$ $\rightarrow V \times A \rightarrow(e)$, where $P$ is maximal periodic, $A$ is abelian, discrete, and aperiodic, and $V$ is isomorphic to $R^{n}$ for some $n$ [10, Theorem (3.16)]. Some condition on $G$ like type I-ness is necessary in the following result.

(1.1) Proposition. Let $G$ and $N$ be as above. If $[\pi] \in \hat{G}$ then the set $\hat{G}_{\pi, N}=\left\{[\gamma] \in \hat{G}: \gamma_{N}\right.$ is quasi-equivalent to $\left.\pi_{N}\right\}$ equals $\left[(G / N)^{\wedge} \otimes \pi\right]$.

Proof. Suppose $[\gamma] \in \hat{G}_{\pi, N}$, and let $[\rho] \in \hat{N}$ be an element of the G-orbit $\theta_{\pi}$ in $\hat{N}$ which is determined by $\pi[1]$. Ket $K(\rho)$ be the stability group of $\rho$ under $G$ 's action on $\hat{N}$ by inner automorphisms. By the Mackey theory there is a $[\sigma] \in K(\rho)^{\wedge}$ so that the induced representation $\operatorname{Ind}_{K(\rho)}^{G}(\sigma)$ is equivalent to $\pi$. Moreover, $\sigma$ may be constructed as follows: There is a multiplier $\omega$ on $K(\rho) / N$ such that $\rho$ extends to an $\omega$ representation of $K(\rho)$. Let $\tilde{\rho}$ be any such extension of $\rho$; then there exists an irreducible $\bar{\omega}$ representation $\beta$ of $K(\rho) / \mathrm{V}$ such that $\beta^{\prime} \otimes \tilde{\rho} \simeq \sigma$. Because of our hypothesis on $G$ and $N$, all primary $\bar{\omega}$ representations of $K(\rho) / N$ are type I [17, Theorem 8.4], and it follows from [3, Theorem (3.3)], together with [3, Theorem (3.1)], that all the irreducible $\omega^{-1}$ representations of $K(\rho) / N$ are on the form $\beta \otimes \chi$ where $\chi$ is some character of $K(\rho) / N$. From this it follows that every $[\gamma] \in \hat{G}_{\pi, N}$ is induced from representations of the form $\chi^{\prime} \otimes \beta^{\prime} \otimes \tilde{\rho}=\chi^{\prime} \otimes \sigma$. Observe now that

$$
\operatorname{Ind}_{K(\rho)}^{G}\left(\chi^{\prime} \otimes \sigma\right) \simeq \tilde{\chi}^{\prime} \otimes \operatorname{Ind}_{K(\rho)}^{G}(\sigma)
$$

where $\tilde{\chi}$ is any extension of the character $\chi$ to $G / N$ and $\tilde{\chi}^{\prime}$ denotes the lift of $\tilde{\chi}$ to $G$ [7, Lemma 4.2]. This completes our proof.(5)

The following result is well known and will be helpful when combined with (1.1).

(1.2) LeммA. Let $G$ be a separable locally compact group and let $K$ be a compact normal subgroup. Then $\hat{G}_{\pi, K}=\left\{[\gamma] \in \hat{G}: \gamma_{K}\right.$ is quasi-equivalent to

(5)A different proof of (1.1) was pointed out to the author by J. Brezin. Brezin's proof also uses the Mackey theory. 
$\left.\pi_{K}\right\}$ is an open and closed subspace of $\hat{G}$.

Proof. If [ $\pi] \in \hat{G}$ let $\theta_{\pi}$ be the $G$-orbit in $\hat{K}$ associated to [ $\pi$ ]; the map $p:[\pi] \mapsto \theta_{\pi}, \hat{G} \rightarrow \hat{K} / G$ is continuous [6, Lemma 3] (the orbit space $\hat{K} / G$ is given the quotient topology induced from $\hat{K}$ ). Since $K$ is compact, $\hat{K}$ and $\hat{K} / G$ are discrete. The result now follows from the fact that $\hat{G}_{\pi, K}=p^{-1}\left(\left\{\theta_{\pi}\right\}\right)$. Q.E.D.

Let $G$ be a locally compact separable type I group and suppose that $K$ is a compact normal subgroup of $G$ such that $G / K$ is abelian.(6) Let $\pi \in \operatorname{Irr}(G)$ be fixed but arbitrary.

There is a natural map $f_{\pi}$ of $\widehat{G / K}$ onto $[\widehat{G / K} \otimes \pi]$ given by $\alpha \mapsto\left[\alpha^{\prime} \otimes \pi\right]$. We let $M_{\pi}=[\widehat{G / K} \otimes \pi]$ and provide $M_{\pi}$ with the topology induced from $\hat{G}$. We may define a natural group structure on $M_{\pi}$ by letting

$$
\left[\alpha^{\prime} \otimes \pi\right] *\left[\beta^{\prime} \otimes \pi\right]=\left[\alpha^{\prime} \beta^{\prime} \otimes \pi\right], \quad \alpha, \beta \in \widehat{G / K} .
$$

It is easy to check that the product $*$ is well defined. Moreover, $G \widehat{G / K}$ acts on $M_{\pi}$ as a locally compact Hausdorff topological transformation group. Indeed, the map $\left(\alpha,\left[\beta^{\prime} \otimes \pi\right]\right) \mapsto\left[\alpha^{\prime} \beta^{\prime} \otimes \pi\right]$ from $\widehat{G / K} \times M_{\pi}$ onto $M_{\pi}$ is continuous, and the map $\alpha \mapsto\left(\left[\beta^{\prime} \otimes \pi\right] \mapsto\left[\alpha^{\prime} \beta^{\prime} \otimes \pi\right]\right)$ is a homomorphism of $\widehat{G / K}$ into the group of homeomorphisms of $M_{\pi}$, and the map $\left[\alpha^{\prime} \otimes \pi\right] \mapsto\left[\alpha_{0}^{\prime} \alpha^{\prime} \otimes \pi\right]$ is a homeomorphism for each $\alpha_{0} \in \widehat{G / K}$. Since $M_{\pi} / \widehat{G / K}$ consists of one point, it is certainly $T_{0}$ and it follows from J. Glimm [8, Theorem 1], that the map $\alpha \cdot$ $G(\pi) \mapsto\left[\alpha^{\prime} \otimes \pi\right]$ from $G / K / G(\pi)$ onto $M_{\pi}$ is a homeomorphism, where $G(\pi)=$ $\left\{\alpha \in G \widehat{G / K}: \alpha^{\prime} \otimes \pi \simeq \pi\right\}$ is the stability group of $[\pi]$. Here the type $I$ hypothesis was needed. Since the quotient map $\widehat{G / K} \rightarrow G / K / G(\pi)$ is open, it follows that $f_{\pi}$ is open and $M_{\pi}$ is Hausdorff. It also follows that $M_{\pi}$ is a topological group with the product * defined above, and $M_{\pi}$ may be identified with the quotient group $G \widehat{G / K} / G(\pi)$. Thus $M_{\pi}$ is even $T_{4}$ by abelian theory [11]. We have almost proved the following theorem.

(1.3) THEOREM. Let $G$ be a separable locally compact group with a compact normal subgroup $K$ such that the factor group $G / K$ is abelian. Then the following statements are equivalent.

(1) $G$ is of type $I$.

(2) $\hat{G}$ is the disjoint union of locally compact abelian topological $T_{4}$ groups $[\widehat{G / K} \otimes \pi]$ which are open in $\hat{G}$.

(3) $\hat{G}$ is $T_{4}$.

(6) The following arguments hold whenever $[\widehat{G / K} \otimes \pi]$ is closed in $\hat{G}$ since $[\widehat{G / K} \otimes \pi]$ is then locally compact. We shall only need to consider compact $K$. 
Proof. $(1) \Rightarrow(2)$. If $G$ is type I then $\hat{G}_{\pi, K}$ equals $[\widehat{G / K} \otimes \pi]$ for all $[\pi] \in$ $\hat{G}$, by (1.1). Hence the implication follows from (1.2) and the arguments above. (2) $\Rightarrow(3) \Rightarrow(1)$ is obvious. Q.E.D.

Note. As observed in [16] a separable type $I[F C]^{-}$group is $[F D]^{-}$. Moreover, a group satisfies the hypothesis of (1.3) iff it is $[F D]^{-}$. Hence it is clear that Theorem (1.3) is a result about $[F C]^{-}$groups.

In this connection we note that J. Liukkonen [16, Theorem 3.6], proved that separable $[F C]^{-}$groups of type I have Hausdorff duals. We feel that our proof of this result is simpler than the one given in [16], but more important, Theorem (1.3) reduces the study of the duals of such groups to the study of abelian character groups. This will be needed in the proofs of our subsequent results.

The following characterizations of connected components (c.c.'s) will also be useful (see the proof of (2.1)).

(1.4) Proposition. Let $G$ be a separable $[F C]^{-}$group of type I, and let $[\pi] \in \hat{G}$. Then the connected component of $[\pi]$ is $[\widehat{G / P} \otimes \pi]^{-}$where $P$ denotes the periodic subgroup of $G$.

Proof. If $G$ is of type I we may choose a compact normal subgroup $K$ such that $G / K$ is abelian (see the note above). Let $[\pi] \in \hat{G}$. Since $[\widehat{G / K} \otimes \pi]$ is open and closed, it suffices to argue in this subspace of $\hat{G}$. Let

$$
f_{\pi}: \alpha \mapsto[\alpha \otimes \pi], \widehat{G / K} \rightarrow[\widehat{G / K} \otimes \pi]
$$

be the canonical map. As we have seen (in front of Theorem (1.3)) $f_{\pi}$ is an open and continuous homomorphism between abelian groups. Hence, since $[\widehat{G / K} \otimes \pi]$ is $T_{0}$ with the relative topology from $\hat{G}$, the c.c. of $[\pi]$ in $[\widehat{G / K} \otimes \pi]$ equals $\overline{f_{\pi}(C)}$ where $C$ is the c.c. of 1 in $\widehat{G / K}[11,7.12]$. Now the periodic subgroup of $G / K$ equals $P / K$ so by abelian theory $[11,24.17] C$ equals $(G / K / P / K)^{\wedge} \approx(G / P)^{\wedge}$. Thus the c.c. of $[\pi]$ is $[\widehat{G / P} \otimes \pi]^{-}$. Q.E.D.

In case the periodic group $P$ is compact $\hat{G}_{\pi, P}$ is closed for all $[\pi] \in \hat{G}$ by (1.2). Moreover, $[\widehat{G / P} \otimes \pi]$ equals $\hat{G}_{\pi, P}(1.1)$. Hence we have

(1.5) Corollary. Let $G \in[F C]^{-}$be separable and of type I. Assume that the periodic group $P$ is compact. If $[\pi] \in \hat{G}$ then its connected component is $[\widehat{G / P} \otimes \pi]$.

2. Duality theory for $[F C]^{-}$groups. We are now in a position to make an analysis, like we did for the character space $X(G)$ in [23], of the dual space $\hat{G}$ for separable $[F C]^{-}$groups. First we observe that if $K$ is a compact normal subgroup of $G$ and $[\pi] \in \hat{G}$, then the restriction $\pi_{K}$ is equivalent to a direct sum 
$m \cdot \bigoplus \omega$ where $m$ is some (at most countable) cardinal number and the sum is taken over some $\hat{G}$-orbit in $\hat{K}$ under the action of $G$ on $\hat{K}$ by inner automorphisms. Observe also that $\hat{G}_{\pi, K}$ consists of all elements in $G$ which lie over the same $G$-orbit as $[\pi]$. Using this together with Theorem (1.3) and the fact that $C_{\pi}=$ $[\widehat{G / P} \otimes \pi]^{-}$for type I groups, we shall prove results analogous to the ones in $\S \S 2$ and 3 of [23]. We shall also discuss square-integrable representations.

(2.1) Proposition. Let $G$ be a separable $[F C]^{-}$group. Then the following results hold

(1) $\hat{G}$ is connected iff $G$ is aperiodic.

(2) Let $G$ be type I. Then $\hat{G}$ is totally disconnected iff $G$ is periodic.

(3) If $G$ is type I and the periodic subgroup $P$ is finite then $\hat{G}$ has finitely many connected components. If $G \in[F I A]^{-}$and $\hat{G}$ has finitely many connected components, then the periodic subgroup $P$ of $G$ is finite.

(4) Let $G$ be type I. $\hat{G}$ is locally Euclidean iff $G$ is compactly generated.

Proof. (1) If $\hat{G}$ is connected then the component $C_{\iota}$ of the trivial representation $\iota$ constitutes all of $\hat{G}$. But $C_{l}$ is easily seen to equal $\widehat{G / P}$ even in the non-type I case. By the Gel'fand-Raikov theorem, $P=(e)$ and $G$ is aperiodic. Conversely, if $G$ is aperiodic then $G$ is abelian [10]. Hence $\hat{G}$ is connected by abelian theory.

(2) Assume $\hat{G}$ is totally disconnected and let $P$ be the periodic subgroup of $G$. Then $\widehat{G / P}=C_{\iota}$ is trivial, hence $G=P$. Conversely, if $G$ is of type $I$ and $G=P$, let $[\pi] \in \hat{G}$. By (1.4) the connected component of $[\pi]$ equals $[\widehat{G / P} \otimes \pi]^{-}=[\pi]^{-}=[\pi]$ since by hypothesis points are closed in $\hat{G}(1.3)$. Hence $\hat{G}$ is totally disconnected.

(3) Let $G$ be type $I$ and suppose $P$ is finite. Then there is only a finite number of $G$-orbits in $\hat{P}$, and hence there is only a finite number of different connected components $[\widehat{G / P} \otimes \pi]=\hat{G}_{\pi, P}(1.5)$. Conversely, let $G \in[F I A]^{-}$and suppose that $\hat{G}$ has only a finite number of connected components $C_{\pi}$. Then each $C_{\pi}$ is open and closed in $\hat{G}$. Let $\#:[\pi] \mapsto \pi$ be the canonical map of $\hat{G}$ onto $X(G)[18,5.1$ and 5.8]. \# is an open and continuous map; hence it maps the open $C_{\pi}$ onto the connected component $C_{\pi}$ of the character $\pi^{\#}$ in $X(G)$. Therefore $\mathfrak{X}(G)$ has only a finite number of connected components, and it follows from [23, Proposition (2.10)], that the periodic subgroup $P$ of $G$ is finite.

(4) Let $G$ be type I and fix a compact normal subgroup $K$ of $G$ such that $G / K$ is abelian. In view of Theorem (1.3), $\hat{G}$ is locally Euclidean iff $\widehat{G / K}$ is locally Euclidean. By abelian theory [19, Corollary 1 to Theorem 2.5], the last assertion holds iff $G / K$ is compactly generated, which is equivalent to $G$ being compactly generated. Q.E.D.

We say that $G$ is an (L) group if every compact subset $F$ of $G$ is contained 
in an open compactly generated normal subgroup $N$ of $G$ such that $G / N$ is aperiodic [20].

(2.2) Proposition. (1) Let $G \in[F C]^{-}$be separable and type I. Then $\hat{G}$ is locally connected if $G$ is an (L) group.

(2) Let $G$ be an $[F D]^{-}$group. Then $G$ is an (L) group if $G$ is locally connected.

Proof. (1) Suppose $G$ is an (L) group. Arguing as in the proof of [23, Proposition (3.4)] (with $B=I(G)$ ), we see that the periodic subgroup $P$ is compact. If $G$ is type $I$ each connected component $C_{\pi}$ is of the form $[\widehat{G / P} \otimes \pi$ ] = $\hat{G}_{\pi, P}$ and is open and closed in $\hat{G}$ since $P$ is compact, (1.1) and (1.2). Also, the canonical map $\widehat{G / P} \rightarrow[\widehat{G / P} \otimes \pi]$ is open and continuous so that it suffices to prove that $\widehat{G / P}$ is locally connected. Since our proposition holds for abelian groups (K. Fan [5]), the problem is reduced to showing that $G / P=\mathbf{R}^{n} \times A$ (where $A$ is discrete abelian and aperiodic) is an (L) group if $G$ is an (L) group, and this was proved in the last part of [23, Proposition (3.3)].

(2) Conversely, suppose $G \in[F D]^{-}$and $\hat{G}$ is locally connected. Let $C$ denote the intersection of all compact invariant neighborhoods of $e$ in $G$. It is easy to check that $G$ is an (L) group whenever $G / C$ is so (see [23, proof of Proposition (3.5)]). Let us show that $G / C$ is an (L) group. Since $C$ is compact, $\widehat{G / C}$ is naturally embedded in $\hat{G}$ as an open subspace; hence $\widehat{G / C}$ is locally connected. Now $G / C \in[F I A]^{-}$so we may apply the open and continuous surjection \#: $\widehat{G / C} \rightarrow \mathfrak{X}(G / C)$ (see the proof of (2.1)). Thus $\mathfrak{X}(G / C)$ is locally connected and it follows from [23, Proposition (3.5)], that $G / C$ is an (L) group. Q.E.D.

Finally, we turn to the question of square-integrability for representations of $[F C]^{-}$groups. We say that $[\pi] \in \hat{G}$ is square-integrable if all the coordinate functions $x \mapsto(\pi(x) u, u)$ of $\pi$ are in $L^{2}(G)$ w.r.t. left Haar measure on $G$. If $G$ is unimodular, $[\pi]$ is square-integrable iff $\pi$ occurs in the left regular representation of $G$ as a subrepresentation [4]. It is known that $[F C]^{-}$groups are unimodular.

(2.3) THEOREM. Let $G$ be a separable and type I $[F C]^{-}$group, and fix $[\pi] \in \hat{G}$. Then the following three statements are equivalent.

(1) $[\pi]$ is square-integrable.

(2) There is a compact normal subgroup $K$ of $G$ such that $G / K$ is abelian and $[\widehat{G / K} \otimes \pi]=[\pi]$.

(3) $[\pi]$ is open in $\hat{G}$.

Each of the above statement implies the following.

(4) $[\widehat{G / P} \otimes \pi]=[\pi]$, where $P$ is the periodic subgroup of $G$.

Proof. (1) $\Rightarrow(2)$. Let $[\pi] \in \hat{G}$ be square-integrable, and let $\rho$ be an irreducible subrepresentation of $\pi_{K}$. If $G(\rho)$ is the stability group of $\rho$ under 
$G$ 's action by inner automorphisms, we know from the Mackey theory that there is a multiplier $\omega: G(\rho) / K \times G(\rho) / K \rightarrow \mathrm{T}$ such that $\rho$ extends to an $\omega$-representation $\tilde{\rho}$ of $G(\rho)[17]$; moreover there is an $\omega^{-1}$ representation $\sigma^{\prime}$ of $G(\rho)$ (which is the identity operator on $K$ ) such that $\pi$ is induced from the ordinary representation $\tilde{\rho} \otimes \sigma^{\prime}$. Let $\sigma$ be the representation of $G(\rho) / K$ given by $\sigma^{\prime}$, and set $S_{\omega}=\{x \in G(\rho): \omega(x, y)=\omega(y, x)$, all $y \in G(\rho)\}$. We have $\sigma=\alpha \otimes \gamma^{\prime}$ where $\alpha$ is a character of $G(\rho) / K$ and $\gamma$ is an $\omega^{-1}$ representation of $G(\rho) / S_{\omega}$ [3, Theorem 3.1] (replace $\omega$ by a similar multiplier, if necessary). Hence $\sigma^{\prime}$ equals $\alpha$ times the identity operator on $S_{\omega}$. Using [14, Corollary 11.1] we see that $\sigma^{\prime}$ is a square-integrable $\omega^{-1}$ representation, and since $\sigma^{\prime}(s)=\alpha(s) I$ on $S_{\omega}$ it is clear that $S_{\omega}$ is compact. $S_{\omega}$ is also normal in $G$, since $G / S_{\omega}$ is abelian. Moreover, the type I hypothesis on $G$ implies that $\omega$ is a type I multiplier. Hence $G(\rho) / S_{\omega}$ has the unique irreducible $\omega^{-1}$ representation $\gamma[3$, Theorem 3.3]. Hence

$$
\left(\tilde{\rho} \otimes \sigma^{\prime}\right) \otimes \beta=\tilde{\rho} \otimes\left(\alpha \otimes \gamma^{\prime}\right)^{\prime} \otimes \beta \simeq \tilde{\rho} \otimes \sigma^{\prime},
$$

for all characters $\beta$ of $G(\rho)$ which are one on $S_{\omega}$. Using the fact that $\operatorname{Ind}_{G(\rho)}^{G}\left(\tilde{\rho} \otimes \sigma^{\prime}\right) \otimes \tilde{\chi} \simeq \operatorname{Ind}_{G(\rho)}^{G}\left(\tilde{\rho} \otimes \sigma^{\prime} \otimes \chi\right)$ where $\chi$ is any character of $G(\rho)$ equal to one on $K$ and $\tilde{\chi}$ is any extension of $\chi$ to $G$ [7, Lemma 4.2], we now have

$$
\pi \otimes \tilde{\chi} \simeq \operatorname{Ind}_{G(\rho)}^{G}\left(\tilde{\rho} \otimes \sigma^{\prime} \otimes \chi\right) \simeq \operatorname{Ind}_{G(\rho)}^{G}\left(\tilde{\rho} \otimes \sigma^{\prime}\right) \simeq \pi
$$

for any character $\tilde{\chi}$ of $G$ which is one on $S_{\omega}$, and where $\chi$ denotes its restriction to $G(\rho)$. Hence $[\widehat{G / S} \omega \otimes \pi]=[\pi]$, and (2) follows.

(2) $\Rightarrow$ (3). If $[\widehat{G / K} \otimes \pi]=[\pi]$ for some compact $K$ then $[\pi]$ is open in $\hat{G}$ by (1.1) and (1.2).

(3) $\Rightarrow(1)$. If $[\pi]$ is open in $\hat{G}$ then $[\pi]$ is square-integrable by $[4,19.8 .5]$, since $\mu([\pi])>0$, where $\mu$ denotes the Plancherel measure for $G$. (It is known that $[F C]^{-}$groups are amenable [15], i.e., $\hat{G}=\hat{G}_{r}$ where $\hat{G}_{r}$ denotes the reduced dual of $G$. We needed this fact here to ensure that $[\pi] \in \hat{G}_{r}$.)

$(2) \Rightarrow(4)$. Suppose there is a compact normal subgroup $K$ of $G$ such that $G / K$ is abelian and $[G / K \otimes \pi]=[\pi]$. Then $K \subset P$ and we have

$$
[\pi] \in[\widehat{G / P} \otimes \pi] \subset[\widehat{G / K} \otimes \pi]=[\pi]
$$

so that $[\pi]=[\widehat{G / P} \otimes \pi]$. Q.E.D.

ADDED IN PROOF. The author has extended Theorem (2.3), (3), to separable [IN]-groups (Isolated points in duals of certain locally compact groups, to appear in Math. Ann.). 


\section{REFERENCES}

1. L. Auslander and C. C. Moore, Unitary representations of solvable Lie groups, Mem. Amer. Math. Soc. No. 62 (1966), 199 pp. MR 34 \#7723.

2. L. Baggett, A separable group having a discrete dual space is compact, J. Functional Analysis 10 (1972),131-148. MR 49 \#10816.

3. L. Baggett and A. Kleppner, Multiplier representations of abelian groups, J. Functional Analysis 14 (1973), 299-324.

4. J. Dixmier, Les $C^{*}$-algèbres et leurs représentations, Gauthier-Villars, Paris, 1964. MR 30 \#1404.

5. K. Fan, On local connectedness of locally compact Abelian groups, Math. Ann. 187 (1970), 114-116. MR 41 \#7022.

6. J. M. G. Fell, $A$ new proof that nilpotent groups are CCR, Proc. Amer. Math. Soc. 13 (1962), 93-99. MR 24 \#A3238.

7. - Weak containment and induced representations of groups, Canad. J. Math. 14 (1962), 237-268. MR 27 \#242.

8. J. G. Glimm, Locally compact transformation groups, Trans. Amer. Math. Soc. 101 (1961), 124-138. MR 25 \#146.

9. S. Grosser, R. Mosak and M. Moskowitz, Duality and harmonic analysis on central topological groups. I, II, Nederl. Akad. Wetensch. Proc. Ser. A 76 = Indag. Math. 35 (1973), 65-77, 78-91. MR 49 \#5225a, b.

10. S. Grosser and M. Moskowitz, Compactness conditions in topological groups, J. Reine Angew. Math. 246 (1971), 1-40. MR 44 \#1 766.

11. E. Hewitt and K. A. Ross, Abstract harmonic analysis, Vol. I, Grundlehren math. Wiss., Band 115, Springer-Verlag, Berlin; Academic Press, New York, 1963. MR 28 \#158.

12. E. Kaniuth, Zur harmonischen Analyse klassenkompakter Gruppen, Math. Ż. 110 (1969), 297-305. MR 41 \#8591.

13. E. Kaniuth and G. Schlichting, Zur harmonischen Analyse klassenkompakter Gruppen. II, Invent. Math. 10 (1970), 332-345. MR 47 \#5521.

14. A. Kleppner and R. L. Lipsman, The Plancherel formula for group extensions. I, Ann. Sci. École Norm. Sup. (4) 5 (1972), 459-516. MR 49 \#7387.

15. H. Leptin, Zur harmonischen Analyse klassenkompakter Gruppen, Invent. Math. 5 (1968), 249-254. MR 38 \#2257.

16. J. L. Liukkonen, Dual spaces of groups with precompact conjugacy classes, Trans. Amer. Math. Soc. 180 (1973), 85-108. MR 47 \#6937.

17. G. W. Mackey, Unitary representations of group extensions. 1, Acta Math. 99 (1958), 265-311. MR 20 \#4789.

18. R. D. Mosak, The $L^{1}$ - and $C^{*}$-algebras of $[F I A]_{\bar{B}}$ groups, and their representations, Trans. Amer. Math. Soc. 163 (1972), 277-310. MR 45 \#2096.

19. M. Moskowitz, Homological algebra in locally compact abelian groups, Trans. Amer. Math. Soc. 127 (1967), 361-404. MR 35 \#5861.

20. L. S. Pontryagin, Continuous groups, 2nd ed., GITTL, Moscow, 1954; English transl., Topological groups, Gordon and Breach, New York, 1966. MR 17, 171; 34 \#1439.

21. L. C. Robertson, A note on the structure of Moore groups, Bull. Amer. Math. Soc. 75 (1969), 594-599. MR $39 \# 7027$.

22. I. Schochetman, Topology and the duals of certain locally compact groups, Trans. Amer. Math. Soc. 150 (1970), 477-489. MR 42 \#422.

23. T. Sund, Duality theory for locally compact groups with precompact conjugacy classes. I: The character space, Trans. Amer. Math. Soc. 211 (1975), 185-202. 